\title{
URLLC and eMBB coexistence in unlicensed spectrum: a preemptive approach
}

\author{
Ayat Zaki-Hindi ${ }^{1,2}$, Salah-Eddine Elayoubi ${ }^{3}$, Tijani Chahed ${ }^{2}$ \\ ${ }^{1}$ Orange Labs, 44 Avenue de la Republique, 92320 Chatillon, France \\ ${ }^{2}$ Institut Polytechnique de Paris; Telecom SudParis; UMR CNRS SAMOVAR, 19 Place Marguerite Perey, 91120 Palaiseau, France \\ ${ }^{3}$ CentraleSupelec, 3 Rue Joliot Curie, 91190 Gif-sur-Yvette, France \\ ayat.zakihindi@orange.com, salaheddine.elayoubi@centralesupelec.fr, tijani.chahed@telecom-sudparis.eu
}

\begin{abstract}
We study in this paper the coexistence of Ultra Reliable Low Latency Communications (URLLC) and enhanced Mobile Broadband (eMBB) services in unlicensed spectrum for the uplink transmission in a 5G smart-factory scenario. We first model the medium access for both services coexisting in unlicensed spectrum and evaluate their performance metrics: high reliability and stringent delay budget for URLLC and throughput for eMBB. The results show that URLLC requirements cannot be met even for low eMBB traffic load. In order to cope with this, we explore a preemptive approach where URLLC packets are transmitted with high power when their delay approaches the delay constraint, increasing their chance of being successfully received. This approach enhances URLLC performance, with a little impact on that of eMBB. We finally show that with a good calibration of some system parameters can lead to an optimal performance for both services.
\end{abstract}

Keywords: URLLC, eMBB, unlicensed spectrum.

\section{INTRODUCTION}

Many challenges are present in 5G wireless networks mainly because of the high demand and the scarcity of resources. The major part of $5 \mathrm{G}$ traffic comes from enhanced Mobile Broadband (eMBB) which represents the traffic generated by users using Internet services. However there exists another important type of traffic in $5 \mathrm{G}$ which is Ultra-Reliable Low-Latency Communication (URLLC). As its name suggests, URLLC traffic requires high reliability, on the order of $99.999 \%$, within stringent delays, as small as $1 m s$ [1]. Both services can coexist in many scenarios, as in industrial areas where automated machines need to communicate urgent control traffic, while video and data traffic are in the background.

We explore in this paper the usage of cheap unlicensed spectrum in such confined environments, where the deployment of personal access points can be controlled [2]. While the external interference can be controlled, the coexistence between factory-generated eMBB and URLLC traffic is not an easy task.

The coexistence of URLLC and eMBB on the same resources has already been addressed in the licensed spectrum, notably in the downlink. Previous studies for instance propose to prioritize URLLC packets over eMBB [3], or even preempting ongoing eMBB transmissions in order to guarantee the stringent requirements of URLLC [4]. The coexistence of URLLC and eMBB in unlicensed spectrum however was seldom addressed, mainly because of the regulations imposed on transmission in unlicensed spectrum such as medium sensing and transmission power. The increasing demand of resources leads us to discard some of these regulations such as transmission power to adapt for the new requirements.

In this paper, we focus on the study of URLLC and eMBB coexistence in unlicensed spectrum in the uplink. We first study the harmonious coexistence of these services then propose a preemptive approach for URLLC so as it meets its stringent constraints, where a URLLC packet can preempt resources when its delay budget approaches expiration. We show the benefit of the latter scheme in enabling URLLC to meet its reliability and delay requirements while maintaining an acceptable performance for eMBB. This requires also calibrating some system parameters as shown later.

\section{THE HARMONIOUS COEXISTENCE OF URLLC AND EMBB IN UNLICENSED SPECTRUM}

We consider a smart factory where a large number of machines are communicating their URLLC traffic in the uplink to a central server. Alongside, other users use the network for other purposes and generate eMBB traffic.

During a given time interval, eMBB traffic is considered constant with $N_{e}$ saturated sources transmitting traffic following a contention-based medium access procedure, while URLLC arrivals, stemming from $N_{u}$ sources, are assumed to be sporadic with a probability of arrival for each station $q$.

Another important difference between the two types of services is their packet length: URLLC packets are typically very small, on the order of 32 Bytes, compared to eMBB bulky packets, on the order of a couple of thousands Bytes. The transmission duration of URLLC and eMBB packets is denoted by $t_{u}$ and $t_{e}$, respectively.

As stated above, URLLC has strict requirements in terms of reliability and latency, denoted by $R$ and $T$, respectively. If a packet is not transmitted successfully before $T$, then it is discarded. eMBB traffic is not as demanding as URLLC but it still has to achieve a certain throughput, denoted by $S_{t}$, so as to guarantee a certain Quality of Service (QoS).

The procedure of unlicensed medium access is based on medium sensing before transmitting: Listen-Before-Talk (LBT), similar to Wi-Fi and LTE-LAA for instance. Time is discretized with time unit denoted by time slot. When the packet is first generated, it chooses uniformly an integer number denoted by contention window $(\mathrm{CW})$ from 0 to $W_{\max }-1$, 


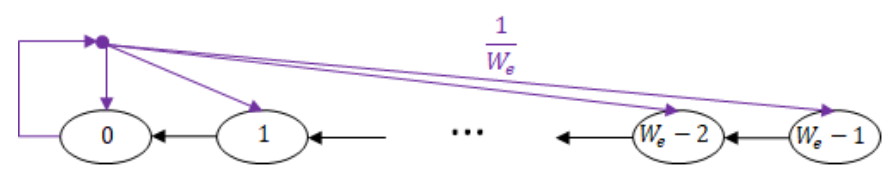

Figure 1: Markov chain model for eMBB

where $W_{\max }$ is the maximum $\mathrm{CW}$ size. After that, the station starts sensing the medium slot by slot to determine whether it is idle or busy. If the medium is sensed idle then CW is decremented by one; else, $\mathrm{CW}$ remains unchanged. The station keeps sensing the medium until $\mathrm{CW}$ reaches zero, in which case it transmits its packet without any sensing. The station knows whether its transmission was successful or not by receiving a positive or negative acknowledgement (Ack/Nack) from the receiver. The absence of acknowledgment after a certain delay is considered as a Nack. In case of Nack, the station repeats the previous procedure until the packet is successfully transmitted or discarded after attaining the maximum number of allowed attempts (also called stages).

We propose deploying LBT cat 3 with fixed $W_{\max }$ in every stage for both services, mainly because it is more adapted to delay-constrained access, and also because it facilitates the analysis. We assume that a collision happens when two or more packets are transmitted at the same time, i.e., colliding packets have $C W=0$ at the same time slot. Other types of interference leading to packet loss are not discussed in this work. We note also that if all stations perform LBT, then after every busy period, an idle time slot is necessarily sensed since all stations have $C W>0$ except for transmitting ones, which leads to decrementing $C W$. A famous model based on Markov chains was proposed by Bianchi in [5] to model the aforementioned procedure, which is adopted in our analysis.

\section{A. eMBB medium access model}

We propose having full-buffer eMBB stations having always a packet to transmit, and it does not matter whether it is an old packet attempt or a new different packet. We further assume that the maximum number of allowed stages is big enough so that the probability of discarding a packet is near zero.

Denoting $W_{\max }=W_{e}$, our corresponding Markov chain of one eMBB station consists of states representing the stochastic process of $\mathrm{CW}$ at time $t$ : $c w(t)$, shown in Figure 1.

This Markov chain is irreducible with positive recurrent states, which means that a stationary distribution $\Pi$ exists and is unique. The stationary probabilities are given by:

$$
\Pi_{W_{e}-j}=\frac{j}{W_{e}} \Pi_{0} \quad \forall j \in\left\{1,2, \ldots, W_{e}\right\}
$$

By applying the normalization condition, which suggests that the sum of all states equals to 1 , we get:

$$
\Pi_{0}=\frac{2}{W_{e}+1}
$$

which is equal to the probability of eMBB transmission denoted by $\tau_{e}$, and depends solely on $W_{e}$.

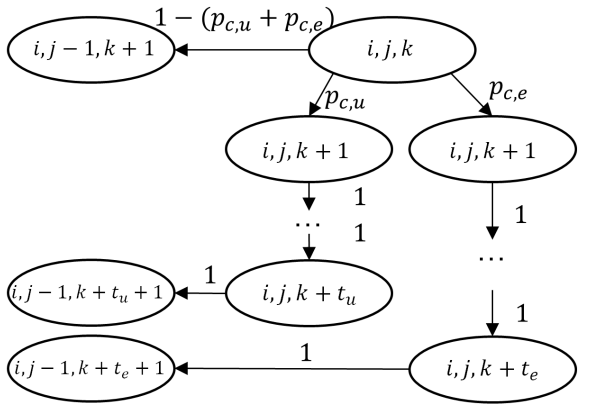

Figure 2: Markov chain for the progress of one state

For eMBB, we choose to measure the QoS by the throughput, defined as the rate of successful message delivery per unit time, described in Equation (1):

$$
S_{e}=\frac{P_{\text {success }}^{e} t_{e}}{P_{i d l e}^{e}+P_{t_{u}} t_{u}+P_{t_{e}} t_{e}}
$$

where:

$$
\begin{gathered}
P_{\text {success }}^{e}=N_{e} \tau_{e}\left(1-\tau_{u}\right)^{N_{u}}\left(1-\tau_{e}\right)^{N_{e}-1} \\
P_{\text {idle }}=\left(1-\tau_{u}\right)^{N_{u}}\left(1-\tau_{e}\right)^{N_{e}} \\
P_{t_{u}}=\left[1-\left(1-\tau_{u}\right)^{N_{u}}\right]\left(1-\tau_{e}\right)^{N_{e}} \\
P_{t_{e}}=1-\left(1-\tau_{e}\right)^{N_{e}}
\end{gathered}
$$

Where $P_{\text {success }}^{e}$ is the probability of having a successful eMBB transmission, $P_{i d l e}$ is the probability of sensing an idle time slot, $P_{t_{u}}$ and $P_{t_{e}}$ are the probability of sensing the medium busy during $t_{u}$ and $t_{e}$ time slots, respectively. We denote by $\tau_{u}$ the probability of transmission for a URLLC station, evaluated in next subsection.

\section{B. URLLC medium access model}

A model of URLLC medium access based on Markov chains was proposed in reference [6], for the case of homogeneous URLLC traffic. This model quantifies the achieved reliability under a delay constraint by calculating the probability of time-out of a packet that enters the system.

URLLC packets are tagged with a delay timer $d$ from the moment of generation, initialized to zero. Stations perform LBT cat 3 and increment $d$ according to the number of sensed time slots; for busy slots, $d$ is either incremented by $t_{u}$ or $t_{e}$ depending on the length of transmission. In this case, we identify each state of the corresponding Markov chain with a triplet $\{s(t), c w(t), d(t)\}$ representing the three stochastic processes: stage, contention window and delay of the packet at time $t$, respectively. Figure 2 illustrates this Markov chain for one state $\{s(t), c w(t), d(t)\}=\{i, j, k\}$, where $p_{c, u}$ and $p_{c, e}$ denote the probability of having an active transmission during $t_{u}$ and $t_{e}$ time slots, respectively. The fact of sensing an idle time slot after every busy period is also illustrated.

We note that $t_{u}$ and $t_{e}$ comprise the time until Ack/Nack reception. We compact all states with transition probabilities of 1 to their final state since it is a deterministic path. Hence, we 


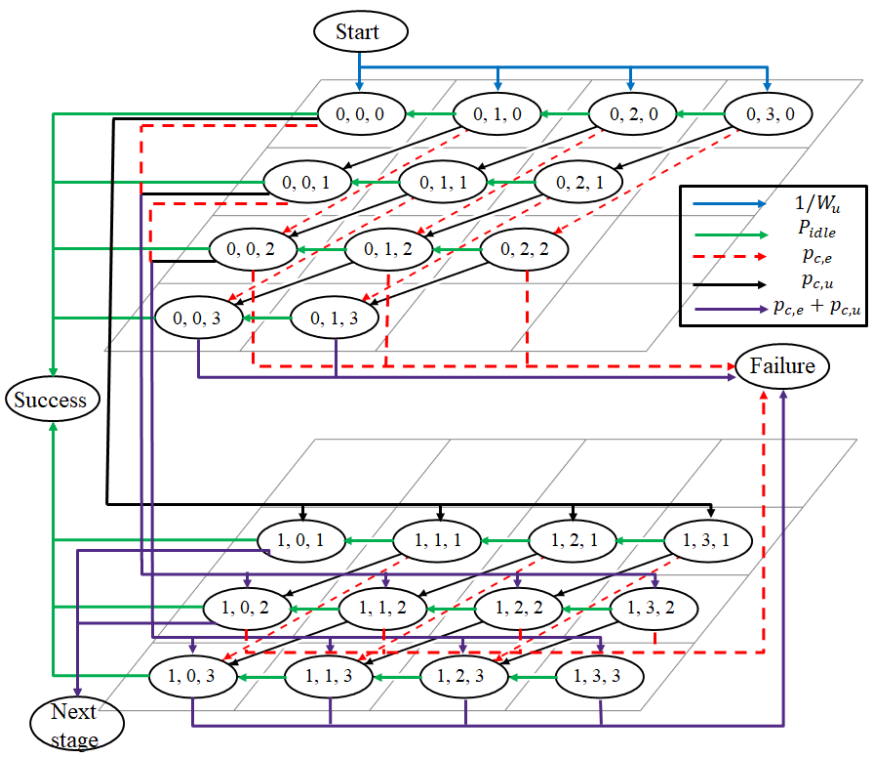

Figure 3: Example of harmonious coexistence Markov chain

have three possible next states to every state, which generates a huge number of states even for small numerical examples. We then propose an approximation to reduce this number by neglecting the one-time-slot increment in $d(t)$ when sensing the medium idle, and keep $t_{u}+1$ and $t_{e}+1$ increments. We further set $t_{e}+1=\alpha\left(t_{u}+1\right)$ and $T=m\left(t_{u}+1\right)$ where $\alpha$ and $m$ are integers. We propose a toy example to illustrate the first two stages of the chain for a given $t_{u}$, having $t_{e}+1=2\left(t_{u}+1\right)$ and $T=3\left(t_{u}+1\right)$. We set $W_{\max }=W_{u}=4$ and get the chain illustrated in Figure 3. In this example, $t_{u}+1 \geq W_{u}$.

States: Start, Success and Failure represent the state of the packet when generated, after a successful transmission and when the delay budget reaches time-out, respectively. We build this transient Markov chain to allow us to calculate the precise probability of failure of URLLC packets by evaluating the hitting probability of state Failure from state Start $h_{\text {Failure }}$. The hitting probability of state Success from state Start $h_{\text {Success }}$ quantifies the reliability. For our analysis, we calculate these probabilities for a packet that already entered the contention phase, hence the hitting probability of Start is $h_{\text {Start }}=1$ and the three probabilities are related by the following relation: $h_{\text {Failure }}+h_{\text {Success }}=h_{\text {Start }}$.

It is worth mentioning that a packet may leave state Start while sensing an ongoing transmission from other stations, hence it has to wait for a fraction of time of $t_{u}$ or $t_{e}$, but this delay is neglected in our analysis for the difficulty of estimating and interpreting these fractions.

In order to calculate $p_{c, u}$ and $p_{c, e}$, we have to render the chain to its recurrent form where states Start, Success and Failure are collapsed to a single state, denoted by Inactive, where in this state the station is idle and is waiting for a new packet to be generated in every time slot with probability $q$. Due to lack of space, we represent all states with index $\{i, j, k\}$ by one state called Contention and illustrate the new chain in

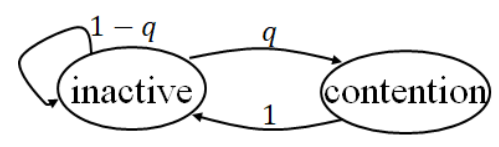

Figure 4: The recurrent form of Markov chain

Figure 4.

We assume $p_{c, u}$ and $p_{c, e}$ are independent from the state of the Markov chain. The chain in Figure 4 is irreducible and its states are positive-recurrent, hence there exist a unique corresponding stationary distribution $\Pi$. We arrange the stationary probabilities of the generalized Markov Chain in a three dimensional matrix denoted by $\Pi$ whose dimensions are $m \times W_{u} \times m$, and every element $\Pi_{i, j, k}$ represents the corresponding state $\{s(t), c w(t), d(t)\}=\{i, j, k\}$.

We initiate the values of the matrix to 0 and start filling the elements in a recursive manner, stage by stage, starting from states with higher $\mathrm{CW}$ and lower delay.

We define the following terms: $A_{1}(i, j, k)$ and $A_{2}(i, j, k)$ that are used frequently in the calculations:

$$
\begin{gathered}
A_{1}(i, j, k)=P_{i d l e} \Pi_{i, W_{u}-j+1, k}+p_{c, u} \Pi_{i, W_{u}-j+1, k-1} \\
A_{2}(i, j, k)=\frac{p_{c, u}}{W_{u}} \Pi_{i-1,0, k-1}+\frac{p_{c, e}}{W_{u}} \Pi_{i-1,0, k-\alpha} \\
A_{3}(i, j, k)=p_{c, u} \Pi_{i, W_{u}-j+1, k-1}+p_{c, e} \Pi_{i, W_{u}-j+1, k-\alpha}
\end{gathered}
$$

The balance equations are hence:

$$
\Pi_{0, W_{u}-1,0}=q \frac{\Pi_{\text {inactive }}}{W_{u}}
$$

$\Pi_{0, W_{u}-j, 0}=q \frac{\Pi_{\text {inactive }}}{W_{u}}+P_{\text {idle }} \Pi_{0, W_{u}-j+1,0}, \quad 2 \leq j \leq W_{u}$

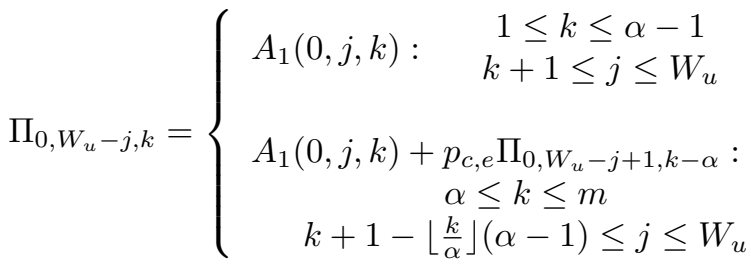

We note that the delay in one stage cannot be less than its number of stage, the first rows in the next stages remain zeros.

For the next stages where: $1 \leq i \leq m$ and $2 \leq j \leq W_{u}$ :

$$
\begin{aligned}
& \Pi_{i, W_{u}-1, k}= \begin{cases}\frac{p_{c, u}}{W_{u}} \Pi_{i-1,0, k-1}: i \leq k \leq i+\alpha-1 \\
A_{2}(i, 1, k): & i+\alpha \leq k \leq m\end{cases} \\
& \Pi_{i, W_{u}-j, k}=\left\{\begin{array}{c}
\frac{p_{c, u}}{W_{u}} \Pi_{i-1,0, k-1}+A_{3}(i, j, k): \\
i \leq k \leq i+\alpha-1 \\
A_{2}(i, j, k)+A_{3}(i, j, k): \quad k=\alpha+i \\
A_{2}(i, j, k)+A_{3}(i, j, k) \\
+p_{c, e} \Pi_{i, W_{u}-j+1, k-\alpha}: \\
i+\alpha+1 \leq k \leq m
\end{array}\right.
\end{aligned}
$$


We apply the normalization condition to calculate the value of $\Pi_{\text {inactive: }}$

$$
\Pi_{\text {inactive }}+\sum_{i=0}^{m-1} \sum_{j=0}^{W_{u}-1} \sum_{k=0}^{m-1} \Pi_{i, j, k}=1
$$

From previous analysis, we deduce the probability of transmission of the URLLC packet denoted by $\tau_{u}$, which corresponds to the probability of being in a state with $C W=0$ and transmitting, calculated from the following formula:

$$
\tau_{u}=\sum_{i=0}^{m-1} \sum_{k=0}^{m-1} \Pi_{i, 0, k}
$$

The term in Equation (2) is a function of $p_{c, u}$ and $p_{c, e}$, which in turn depend on $\tau_{u}$ and the probability of an eMBB transmission $\tau_{e}$, calculated in previous subsection. $p_{c, u}$ corresponds to the probability of having at least one URLLC transmission and no eMBB one, given by:

$$
p_{c, u}=\left[1-\left(1-\tau_{u}\right)^{N_{u}-1}\right]\left(1-\tau_{e}\right)^{N_{e}}
$$

$p_{c, e}$ corresponds to the probability of having at least one eMBB transmission regardless of URLLC ones because they have a smaller duration, given by:

$$
p_{c, e}=1-\left(1-\tau_{e}\right)^{N_{e}}
$$

Equations (2), (3) and (4) are a set of fixed-point equations that cannot be solved analytically and must be solved numerically.

To quantify the reliability, we get back to Figure 3 to calculate $h_{\text {Success }}$, the hitting probability of state Success from state Start. After obtaining all probabilities from the fixed point equations, we use the same stationary probability equations with the obtained values, after replacing $q \Pi_{\text {inactive }}$ with $\Pi_{\text {Start }}=1$ and get:

$$
h_{\text {Success }}=P_{\text {idle }} \sum_{i=0}^{m-1} \sum_{k=0}^{m-1} \Pi_{i, 0, k}
$$

We note that $h_{\text {Failure }}=1-h_{\text {Success }}$.

\section{Numerical evaluation}

We consider the numerical values similar to the ones in latest IEEE 802.11 standards, regarding the time slot duration $T_{s}$, backoff durations $S I F S, D I F S$ and bit rate $R_{b}$. We study the medium access on one channel, where there might be multiple available channels chosen randomly for transmission.

The data packet sizes for URLLC and eMBB including all headers are denoted by $L_{u}$ and $L_{e}$, respectively. $L_{e}$ is chosen as the maximum length of a Wi-Fi packet. We denote the acknowledgment packet size by $L_{a c k}$. The station receives the Ack/Nack after a duration of SIFS then all stations backoff during a period of DIFS before starting to contend again for medium access. The duration $t_{u}$ is then calculated as: $t_{u}=\left\lceil\frac{\left(L_{u}+L_{a c k}\right) R_{b}+S I F S+D I F S}{T_{s}}\right\rceil$, where $\lceil$.$\rceil is the ceiling$ function. $t_{e}$ is similarly calculated for $L_{e}$. We consider that

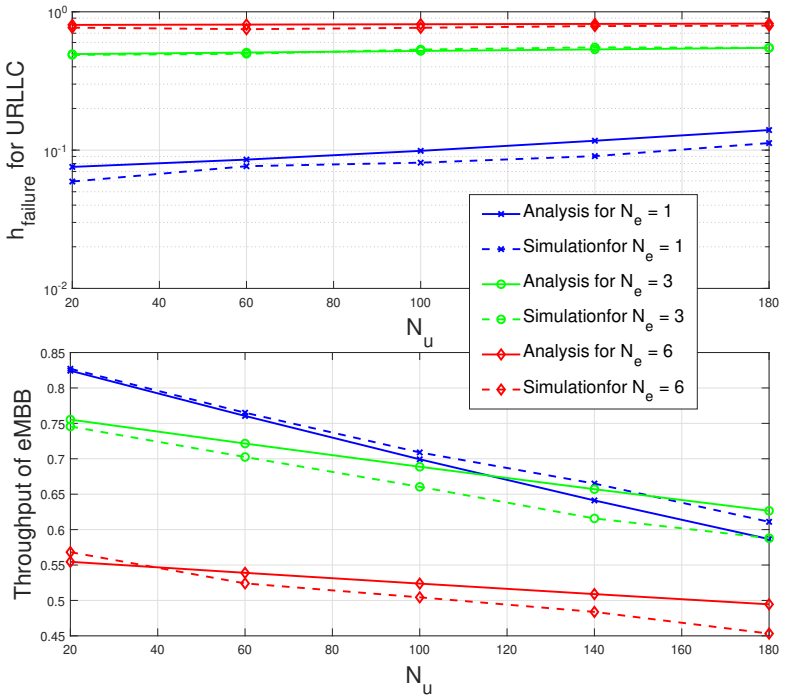

Figure 5: Models validation for the harmonious coexistence

every URLLC station generates a packet every $10 \mathrm{~ms}$ following a Poisson distribution, then the probability of packet arrival $q$ per $T_{s}$ is given approximately by: $q \approx 0.001$. eMBB stations are assumed to be saturated. For URLLC, the reliability constraint is set to $R=1-10^{-5}$ and delay constraint to $1 \mathrm{~ms}$. For eMBB, target normalized throughput is set to $S_{e}=0.7$. Table I shows the values of the system parameters used in the numerical applications.

Table I: Numerical values of the system

\begin{tabular}{|c|c||c|c||c|c|}
\hline$T_{s}$ & $9 \mu s$ & $L_{u}$ & 32 Bytes & $R_{b}$ & 100 Mbps \\
\hline SIFS & $16 \mu s$ & $L_{e}$ & 2312 Bytes & $t_{u}$ & 6 \\
\hline DIFS & $34 \mu s$ & $L_{a c k}$ & 14 Bytes & $t_{e}$ & 27 \\
\hline
\end{tabular}

We validate our analytical models with simulations for different values of $N_{u}$ and $N_{e}$, fixing $W_{u}=W_{e}=10$. In the simulation, we tag eMBB packets with their $\mathrm{CW}$ values in every time slot so they are transmitted when their $\mathrm{CW}$ reaches zero, and for URLLC, we tag only one station to which we are evaluating the probabilities and assume other URLLC packets arrive independently following a Poisson distribution with parameter $q$. URLLC station is tagged with $\mathrm{CW}$ and $d$ and the delay constraint of $1 \mathrm{~ms}$ is verified at every time slot. We count the number of discarded URLLC packets and divide it by the total number of generated packets belonging to one station to obtain $h_{\text {Failure }}$. We also count the number of time slots which contain a successful eMBB transmission and divide it by the length of the simulation in time slots to obtain eMBB throughput.

Figure 5 shows the results obtained by the analytical models and compares them to those obtained through simulation.

We first observe that the results obtained by the models and simulations are very close which validate the former; the difference between analysis and simulations can be as large as $15 \%$ for URLLC and less than $10 \%$ for eMBB. We observe 
also that performance metrics are more affected by $N_{e}$ than $N_{u}$. eMBB's throughput rests acceptable: $S_{e} \geq 0.7$ for small $N_{e}$ values and $N_{u}<100$. However, URLLC's error rate is very far from target value defined in the standards to be around $10^{-5}$, even for small $N_{e}$ and $N_{u}$. This indicates that the harmonious coexistence of eMBB with URLLC is not viable for URLLC, because waiting for large eMBB packets being transmitted leads to time-out of URLLC packets more often.

\section{OPPORTUNISTIC PREEMPTIVE APPROACH}

As illustrated in previous section, the coexistence of URLLC with eMBB degrades the performance of both, especially for URLLC due to its strict constraints on delay and reliability, leading to requiring more resources to guarantee QoS for both. We propose here a new approach to improve the reliability of URLLC, by making use of preemption at the transmission power level. We study the effect of this approach on eMBB performance too, to determine its feasibility.

The preemption scheme we propose is that URLLC and eMBB users coexist harmoniously as explained in previous section, both transmit at the same power. However, when the delay of the transmitted URLLC packet approaches its deadline, i.e., the remaining time budget allows one URLLC packet transmission, then this packet will be transmitted with high power, which increases its chance of being decoded by the receiver. This very packet will be lost only if it is transmitted simultaneously with another high-power URLLC packet. We call this scheme "opportunistic preemption". This scheme violates the process of LBT, which may results in interrupting an ongoing eMBB transmission, decreasing the throughput of eMBB.

\section{A. URLLC medium access model}

We make use of the Markov chain illustrated in Figure 3. We suppose that the URLLC station decides to preempt the channel when the given packet delay reaches $T-t_{u}$ and that it only has one attempt to transmit before time-out, which corresponds to the states with $d(t)=m-1$. We note that states with $d(t)<m-1$ could arrive to the state $d(t)=m-1$ and then to the state Failure when the medium is sensed busy for $t_{e}$ time slots, which is shown in Figure 2.

For simplification, we exchange the state Failure in Figure 3 by a new state which we call Preemption, and from which we have two possible baths to states Failure or Success, in case of collision or no collision with another high-power transmitted packet, respectively. Hence, reliability is increased as a new path to the state Success is created.

The stationary probabilities calculated in last section are still valid here, but for the fixed point equations, we introduce a new probability which is the probability of a preemptive transmission, $\tau_{p}$, defined as the probability that a URLLC station transmits with high power, given by:

$$
\tau_{p}=\sum_{i=0}^{m-1} \sum_{j=0}^{W_{u}-1} \pi_{i, j, m-1}+\sum_{i=0}^{m-1} \sum_{j=0}^{W_{u}-1} \sum_{k=m-\alpha}^{m-2} \pi_{i, j, k}
$$

$\tau_{p}$ is the sum of all states with $d(t)=m-1$ where the second term of the expression corresponds to the implicit states when the medium is busy for $t_{e}$ time slots.

To stay consistent with the analysis in previous section, we denote $\tau_{u}=\tau_{u} *+\tau_{p}$ where:

$$
\tau_{u} *=\sum_{i=0}^{m-1} \sum_{k=0}^{m-1} \pi_{i, 0, k}
$$

We follow the same steps for this analysis as before and obtain the new values of $p_{c, u}$ and $p_{c, e}$.

We denote the new hitting probabilities for states Success and Failure by $h_{\text {Success }}^{\prime}$ and $h_{\text {Failure }}^{\prime}$, respectively. We use the expression in equation (5) to calculate the new probabilities as follows:

$$
\begin{gathered}
h_{\text {Success }}^{\prime}=h_{\text {Success }}+\left(1-\tau_{p}\right)^{N_{u}-1} \times h_{\text {Failure }} \\
h_{\text {Failure }}^{\prime}=1-h_{\text {Success }}^{\prime}
\end{gathered}
$$

And hence we obtain the reliability and loss rate of URLLC for the opportunistic preemption method.

\section{B. eMBB medium access model}

Since eMBB medium access depends only on $W_{e}$, the evaluated model in last section holds. We only evaluate the new value of throughput, which depends on $\tau_{u}, \tau_{e}$ and $\tau_{p}$. The new value of $\tau_{u}=\tau_{u} *+\tau_{p}$ is calculated in previous subsection.

Throughput formula in Equation (1) is still valid, probabilities: $P_{i d l e}, P_{t_{u}}$ and $P_{t_{e}}$ remain the same by substituting the new value of $\tau_{u}$. $P_{\text {success }}^{e}$ becomes:

$$
P_{\text {success }}^{e}=N_{e} \tau_{e}\left(1-\tau_{e}\right)^{N_{e}-1}\left(1-\tau_{u}\right)^{N_{u}}\left(1-\tau_{p}\right)^{N_{u} \alpha}
$$

where it expresses the probability of having one eMBB transmission and no other simultaneous eMBB or URLLC transmissions and no preemption in any part of the $\alpha$ parts of the packet. We considered in this case the preemptive transmissions as additional URLLC stations which transmit with probability $\tau_{p}$ every $t_{u}+1$ time slots.

\section{Numerical evaluation}

We validate the opportunistic preemptive model with simulations, using the same numerical values introduced in Table I. We illustrate the results in Figure 6.

We notice that simulations validate the model, with a limited difference between the curves to $60 \%$ for URLLC and $25 \%$ to eMBB. Actually this difference comes from the difficulty of interpreting the probability of preemption $\tau_{p}$ in simulations, since we consider these transmissions as new URLLC stations, but we do not know their actual probability of transmission. In simulations, we considered this probability equals to $q / 2$, which is not accurate because it depends on $N_{u}$ and $N_{e}$.

We evaluate the preemption rate in the opportunistic approach, given by the percentage of high-power transmissions to the total URLLC transmission rate $\tau_{p} / \tau \times 100$. We obtain the following preemption rates $\{7 \%, 30 \%, 50 \%\}$ numerically 


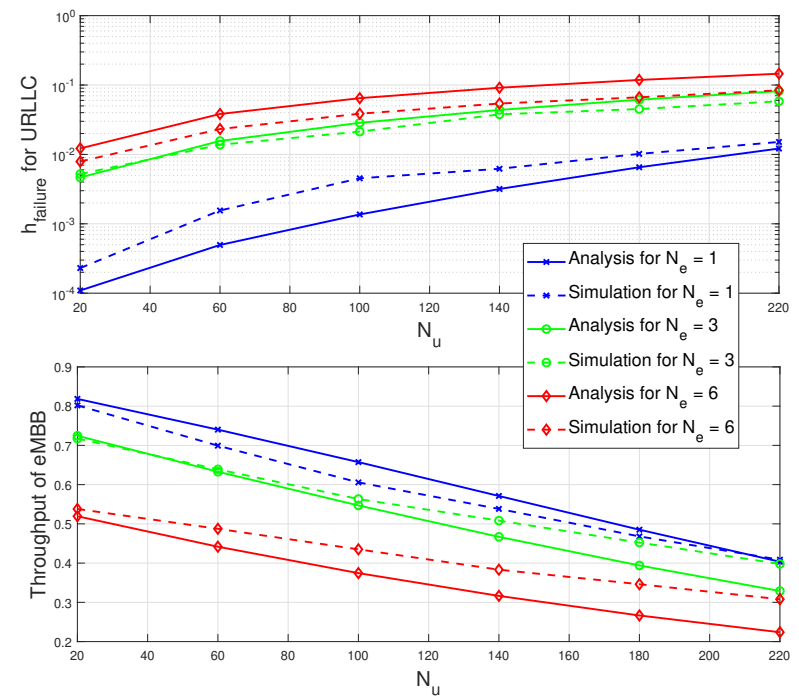

Figure 6: Models validation for opportunistic preemption

for $N_{e}=\{1,3,6\}$, respectively, fixing $N_{u}=100$. These values provide us with insights on LBT violation rate and power consumption. This also justifies the difference between analysis and simulations since we proposed a fixed nonadapted preemption rate for simulations.

Observing Figure 6, eMBB throughput has slightly decreased from the case of harmonious coexistence as predicted from preemption, and it can still be guaranteed for certain, relatively small, $N_{u}$ and $N_{e}$ values, e.g., $N_{e}=1, N_{u} \leq 80$. For URLLC, reliability is highly improved but still does not attain its target of $10^{-5}$ loss rate.

We further perceive the two important parameters $W_{u}$ and $W_{e}$, which play a major role in prioritizing transmission in LBT. As known, reducing the range of $\mathrm{CW}$ for one service gives it more priority over the other, hence, choosing $W_{u}<$ $W_{e}$ may help URLLC packets to reach their target reliability. We illustrate in Figure 7 the effect of different $W_{u}$ and $W_{e}$ values. We illustrate in Figure 7 the cases: $W_{u}=4$ and $W_{u} \leq$ $W_{e}$ for a practical scenario where $N_{e}=3$.

Figure 7 confirms the fact that choosing $W_{u}<W_{e}$ enhances URLLC's reliability, fortunately, this is also shown to enhance eMBB's throughput, because it reduces collisions between eMBB stations. Although this calibration enhances the performance against previous example of $W_{u}=W_{e}=10$, QoS constraints are only verified for a limited range of $N_{u}$ for a given $N_{e}$. From Figure 7, we deduce that setting $W_{u}=4$, $W_{e}=32$ can guarantee the desirable performance for both services when $N_{e}=3$ and $N_{u} \leq 60$, against $N_{u} \leq 20$ from Figure 6.

\section{CONCLUSION}

We studied in this paper the coexistence of URLLC and eMBB services in unlicensed spectrum for the uplink transmission in a $5 \mathrm{G}$ smart-factory scenario. We modeled the medium access for both services and evaluated their performance
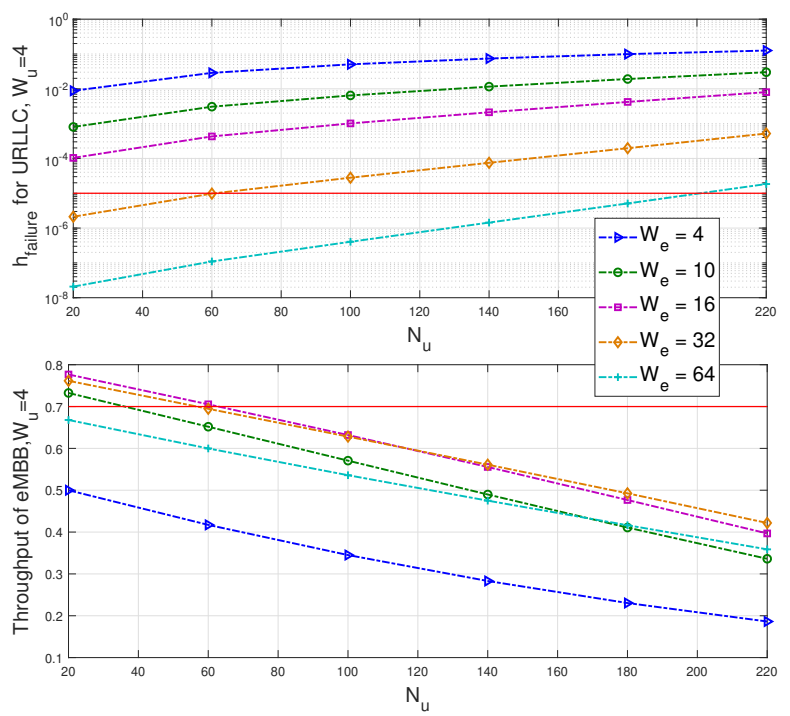

Figure 7: The effect of the maximum contention window size

metrics: reliability and delay for URLLC and throughput for eMBB.

We illustrated numerically the poor performance in terms of reliability for URLLC and proposed an opportunistic preemptive approach in order to prioritize it over eMBB, where URLLC stations transmit with high power when their packet delay approaches the delay budget.

We evaluated the power consumption resulted from the opportunistic approach compared to the no-preemption approach for different loads and showed that higher preemption rates are needed for higher loads.

Finally, we illustrated that by a good calibration for the maximum contention window size for the opportunistic preemptive approach we can verify URLLC and eMBB performance metrics, depending on the number of competing stations.

\section{REFERENCES}

[1] M. Maternia, S. Elayoubi, M. Fallgren, P. Spapis, Y. Qi, D. MartínSacristán, Ó. Carrasco, M. Fresia, M. Payaró, M. Schubert et al., " $5 \mathrm{~g}$ ppp use cases and performance evaluation models," 5G PPP, 2016.

[2] G. J. Sutton, J. Zeng, R. P. Liu, W. Ni, D. N. Nguyen, B. A. Jayawickrama, X. Huang, M. Abolhasan, and Z. Zhang, "Enabling ultra-reliable and lowlatency communications through unlicensed spectrum," IEEE Network, vol. 32, no. 2, pp. 70-77, 2018.

[3] A. Anand, G. De Veciana, and S. Shakkottai, "Joint scheduling of urllc and embb traffic in $5 \mathrm{~g}$ wireless networks," in IEEE INFOCOM 2018-IEEE Conference on Computer Communications. IEEE, 2018, pp. 1970-1978.

[4] A. A. Esswie and K. I. Pedersen, "Opportunistic spatial preemptive scheduling for urllc and embb coexistence in multi-user 5g networks," Ieee Access, vol. 6, pp. 38 451-38 463, 2018.

[5] G. Bianchi, "Performance analysis of the ieee 802.11 distributed coordination function," IEEE Journal on Selected Areas in Communications, vol. 18, no. 3, pp. 535-547, March 2000.

[6] A. Z. Hindi, S. E. Elayoubi, and T. Chahed, "Performance evaluation of ultra-reliable low-latency communication over unlicensed spectrum," ICC 2019 - 2019 IEEE International Conference on Communications (ICC), pp. 1-7, 2019. 